\title{
Nivel de conocimiento sobre medidas de bioseguridad y su empleo por parte de los estudiantes en una Universidad Peruana
}

\author{
Level of knowledge regarding biosafety measures and their use by students in a Peruvian \\ University
}

Victor Humberto Chero Pacheco ${ }^{1}$.

\section{RESUMEN}

Objetivo: Determinar el nivel de conocimiento sobre medidas de bioseguridad y su empleo por parte de los estudiantes frente a situaciones de riesgo en los servicios de atención. Materiales y Métodos: El estudio considerado es de carácter descriptivo y transversal. Se determinó el nivel de conocimiento y empleo de medidas de bioseguridad mediante el cuestionario y uso de fichas de observación. Resultados: El nivel de conocimiento en relación a los conceptos de bioseguridad es alto en un $66,7 \%$ y bajo en un $33,3 \%$. El nivel de conocimiento en relación al uso de barreras es alto en un $83,3 \%$ (uso de guantes), 56,7\% (uso de mascarilla) y 66,7\% (uso de mandil). Es bajo en un $16,7 \%$ (uso de guantes), 43,3\% (uso de mascarilla) y 33,3\% (uso de mandil). El nivel de conocimiento en relación al manejo adecuado de residuos contaminantes es alto en un 56,7\% y bajo en un 43,3\%. El empleo de medidas de bioseguridad muestra que los estudiantes siempre emplean las medidas en un $66,7 \%$ (uso de guantes), $83,3 \%$ (uso de mascarilla), $100 \%$ (uso de mandil) y $50 \%$ (eliminación adecuada de residuos). Emplean a veces las medidas en un 26,7\% (uso de guantes), $16,7 \%$ (uso de mascarilla), y 23,3\% (eliminación adecuada de residuos). Nunca emplean las medidas en un $6,7 \%$ (uso de guantes), y 26,7\% (eliminación adecuada de residuos). Conclusión: Existe un alto nivel de conocimiento sobre las medidas de bioseguridad entre los estudiantes encuestados, así como del uso de barreras y manejo de residuos sólidos.

Palabras clave: conocimiento, medidas, bioseguridad.

\begin{abstract}
Objective: To determine the level of knowledge regarding biosafety measures and their use by students to face risk situations into assistance services. Materials and Methods: Descriptive and transactional survey is considered. The level of knowledge and the use of biosafety measures were measured by using questionnaire and observation record sheet. Results: The level of knowledge in relation to biosafety concepts is high with $66,7 \%$ and low with $33,3 \%$. The level of knowledge regarding the use of barriers is high with $83,3 \%$ (use of gloves), 56,7\% (use of mask) and 66,7\% (use of gown). This level is low with 16,7\% (use of gloves), 43,3\% (use of mask) and 33,3\% (use of gown). The level of knowledge in relation to manipulate appropriately infectious waste is high with $56,7 \%$ and low with $43,4 \%$. The use of biosecurity measures shows that students always use them with $66,7 \%$ (use of gloves), $83,3 \%$ (use of mask), 100\% (use of gown) and 50\% (appropriate disposal of waste). Students sometimes use biosafety measures with 26,7\% (use of gloves), 16,7\% (use of mask), and 23,3\% (appropriate disposal of waste). Students never use biosafety measures with $6,7 \%$ (use of gloves) and 26,7\% (appropriate disposal of waste). Conclusion: There was a high level of knowledge about biosafety measures between survey students, as well as the use of barriers and disposal of solid residues.
\end{abstract}

Key words: knowledge, measures, biosafety.

\footnotetext{
${ }^{1}$ Universidad María Auxiliadora
} 


\section{INTRODUCCIÓN}

Múltiples situaciones de riesgo para la salud son asociadas a la falta de conocimiento en relación a medidas preventivas aplicadas en aquellos ambientes en donde se manipulan agentes específicos que por su naturaleza representan un peligro para la integridad de los individuos que desarrollan procedimientos o actividades en relación a la atención de pacientes; es decir, aquellos procedimientos que implican el riesgo de contacto con elementos capaces de comportarse como vehículos de transmisión de agentes infecciosos. Es necesario desarrollar estrategias que permitan el logro de actitudes y conductas que disminuyan el riesgo de los trabajadores de salud a adquirir infecciones en el medio laboral $(1,2)$.

A pesar de las estrategias preventivas y aquellas medidas de contingencia establecidas y consideradas en el ámbito institucional, la capacidad de reacción de los individuos que en el caso del presente estudio se refiere a estudiantes, se debe considerar un factor importante para la adecuada conducta ante eventos de riesgo en las actividades de atención a pacientes. Es de vital importancia comprender que muchos contaminantes pueden transmitirse por vía aérea y por contacto directo, siendo esto crítico en razón de producir procesos patológicos severos $(3,4)$. Los cuidados que puedan considerarse en el ámbito asistencial; además, serán de suma importancia en la percepción de los pacientes sobre el cuidado de su salud. Es decir, el conocimiento y aplicación de medidas de bioseguridad conseguirá una mejora de la relación personal asistencialpaciente (5).

El objetivo del presente estudio es determinar el nivel de conocimiento sobre medidas de bioseguridad y su empleo por parte de los estudiantes frente a situaciones de riesgo en ambientes de atención clínica.

\section{MATERIALES Y METODOS}

El estudio realizado considera el carácter descriptivo, además de ser un estudio transversal. La población de estudio incluye 30 estudiantes de la Facultad de Ciencias de la Salud de la Universidad María Auxiliadora. La información en relación al nivel de conocimiento sobre medidas de bioseguridad y su empleo se obtuvo en base al conocimiento sobre los conceptos de bioseguridad, barreras protectoras y residuos contaminantes mediante el uso de cuestionarios; además se evaluó el empleo de elementos de barrera y la adecuada disposición de los residuos contaminantes mediante el uso de fichas de observación. Los datos obtenidos fueron procesados empleando sistemas estadísticos que permitirán establecer la frecuencia de los valores en relación a los indicadores antes mencionados.

\section{RESULTADOS}

Con respecto al nivel de conocimiento sobre medidas de bioseguridad y su empleo, se evidencia que el nivel de conocimiento es alto en $66,7 \%$ y bajo en un $33,3 \%$ (Tabla 1 )

Tabla 1. Nivel de conocimiento sobre el concepto de bioseguridad.

\begin{tabular}{|l|c|c|}
\hline \multirow{2}{*}{ Nivel de conocimiento } & \multicolumn{2}{|c|}{ Frecuencia } \\
\cline { 2 - 3 } & $\mathbf{N}^{\circ}$ & $\%$ \\
\hline Alto & 20 & 66,7 \\
Medio & 0 & 0 \\
Bajo & 10 & 33,3 \\
\hline Total & 30 & 100 \\
\hline
\end{tabular}

Con respecto al nivel de conocimiento de uso de guantes es alto en un $83,3 \%$ y bajo en un $16,7 \%$. En el caso del uso de mascarilla, es alto en un $56,7 \%$ y bajo en un $43,3 \%$. Asimismo, en el caso del uso de mandil, es alto en un $66,7 \%$ y bajo en un $33,3 \%$ (Tabla 2).

Tabla 2. Nivel de conocimiento sobre el uso de barreras (guantes, mascarilla, mandil).

\begin{tabular}{|l|c|c|c|c|c|c|}
\hline \multirow{2}{*}{$\begin{array}{l}\text { Nivel } \\
\text { de } \\
\text { conoci- } \\
\text { miento }\end{array}$} & \multicolumn{6}{|c|}{ Uso de barreras } \\
\cline { 2 - 7 } & \multicolumn{2}{|c|}{ Guantes } & \multicolumn{2}{|c|}{ Mascarilla } & \multicolumn{2}{c|}{ Mandil } \\
\cline { 2 - 7 } & $\mathrm{N}^{\circ}$ & $\%$ & $\mathrm{~N}^{\circ}$ & $\%$ & $\mathrm{~N}^{\circ}$ & $\%$ \\
\hline Alto & 25 & 83,3 & 17 & 56,7 & 20 & 66,7 \\
Medio & 0 & 0 & 0 & 0 & 0 & 0 \\
Bajo & 5 & 16,7 & 13 & 43,3 & 10 & 33,3 \\
\hline Total & 30 & 100 & 30 & 100 & 30 & 100 \\
\hline
\end{tabular}

Por otro lado, con respecto al conocimiento de eliminación de residuos contaminantes, es alto en un $56,7 \%$ y bajo en un $43,3 \%$ (Tabla $3)$.

Tabla 3. Nivel de conocimiento sobre eliminación de residuos contaminantes.

\begin{tabular}{|l|c|c|}
\hline \multirow{2}{*}{ Nivel de conocimiento } & \multicolumn{2}{|c|}{ Frecuencia } \\
\cline { 2 - 3 } & $\mathbf{N}^{\circ}$ & $\%$ \\
\hline Alto & 17 & 56,7 \\
Medio & 0 & 0 \\
Bajo & 13 & 43,3 \\
\hline Total & 30 & 100 \\
\hline
\end{tabular}


Finalmente en relación al empleo de medidas de seguridad, los estudiantes siempre emplean las medidas en un $66,7 \%$ (uso de guantes), $83,3 \%$ (uso de mascarilla), $100 \%$ (uso de mandil) y $50 \%$ (eliminación adecuada de residuos), emplean a veces las medidas en un
$26,7 \%$ (uso de guantes), 16,7\% (uso de mascarilla), y 23,3\% (eliminación adecuada de residuos) y nunca emplean las medidas en un $6,7 \%$ (uso de guantes), y 26,7\% (eliminación adecuada de residuos) (Tabla 4).

Tabla4. Aplicación de las medidas de bioseguridad (uso de guantes, mascarilla, mandil, eliminación adecuada de residuos).

\begin{tabular}{|c|c|c|c|c|c|c|c|c|}
\hline \multirow{3}{*}{$\begin{array}{l}\text { Escala de } \\
\text { frecuencia }\end{array}$} & \multicolumn{8}{|c|}{ Empleo de medidas } \\
\hline & \multicolumn{2}{|c|}{ Guantes } & \multicolumn{2}{|c|}{ Mascarilla } & \multicolumn{2}{|c|}{ Mandil } & \multicolumn{2}{|c|}{$\begin{array}{l}\text { Eliminación } \\
\text { de residuos }\end{array}$} \\
\hline & $\mathbf{N}^{\circ}$ & $\%$ & $\mathbf{N}^{\circ}$ & $\%$ & $\mathbf{N}^{\circ}$ & $\%$ & $\mathbf{N}^{\circ}$ & $\%$ \\
\hline Siempre & 20 & 66,7 & 25 & 83,3 & 30 & 100 & 15 & 50 \\
\hline A veces & 8 & 26,7 & 5 & 16,7 & 0 & 0 & 7 & 23,3 \\
\hline Nunca & 2 & 6,7 & 0 & 0 & 0 & 0 & 8 & 26,7 \\
\hline Total & 30 & 100 & 30 & 100 & 30 & 100 & 30 & 100 \\
\hline
\end{tabular}

\section{DISCUSIÓN}

En las especialidades de Salud se hace indispensable el refuerzo constante en relación al conocimiento de medidas que disminuyan o controlen la transmisión de agentes contaminantes durante la interacción con pacientes. A pesar de dicha aclaración, aún se hace evidente el elevado índice del personal que desconoce información básica y relevante respecto a situaciones de riesgo y su control por medio de procedimientos 0 medidas de bioseguridad. Dichos conocimientos deben ser adquiridos durante la formación profesional de los estudiantes y esto será evaluado constantemente con la finalidad de que los parámetros importantes puedan incluirse en la conducta cotidiana de los estudiantes y sean llevados a la práctica durante sus labores de atención clínica. En el presente estudio se determinó que el conocimiento sobre el concepto de bioseguridad es alto en un $66,7 \%$ y bajo en un $33,3 \%$, lo cual indica que en su mayoría poseen conocimientos apropiados respecto a bioseguridad del mismo modo que López R. y López, M. en el estudio "Nivel de conocimiento y prácticas de medidas de bioseguridad de internos de enfermería" concluyeron en su trabajo de investigación, refiriéndose a un nivel bueno y regular dentro de valores apropiados en relación al total de individuos (6).

De acuerdo al conocimiento sobre uso de barreras se determinó que el conocimiento es alto respecto al uso de guantes $(83,3 \%)$, mascarilla $(56,7 \%)$ y mandil $(66,7)$, en comparación a los niveles bajos de conocimiento sobre el uso de guantes $(16,7 \%)$, mascarilla $(43,3 \%)$ y mandil $(33,3 \%)$. Dichos resultados concuerdan con la información de Sangama L. y Rojas R. en el estudio "Nivel de conocimiento y aplicación de medidas de bioseguridad de estudiantes de obstetricia", en donde se indica el nivel de conocimiento alto de acuerdo al uso de barreras, el cual predomina sobre el nivel de conocimiento bajo (7).

Debido al carácter contaminante de los residuos, se considera de mucha importancia el manejo de estos, por lo cual el presente estudio también considera el nivel de conocimiento sobre este parámetro evidenciándose en los resultados un nivel de conocimiento alto representado por el $56,7 \%$ de individuos y un nivel bajo representado por el $43,3 \%$. El resultado muestra concordancia con el estudio de López R. y López M., en donde se concluye respecto al conocimiento sobre residuos un valor alto en relación al buen nivel de conocimiento. Esto puede interpretarse como el interés en identificar las características de dichos elementos residuales lo cual se halla en relación con los procedimientos de cuidado para su disposición y destino.

El conocimiento sobre bioseguridad y los procesos que incluye adquieren su máximo valor cuando son empleados; es decir, puestos en práctica, con la finalidad de reducir y controlar las situaciones de riesgo asociadas a la transmisión de agentes patógenos infecciosos. Por tal motivo, el presente estudio también incluye la evaluación de 
actividades prácticas en razón del uso de barreras de protección y la disposición de residuos contaminantes. De acuerdo a estos parámetros los estudiantes que siempre emplearon los procedimientos adecuadamente representan un alto porcentaje de acuerdo a diferentes actividades. Así tenemos, que siempre emplearon guantes el $66,7 \%$; mascarilla, el 83,3\%; mandil, el $100 \%$ y eliminaron los residuos adecuadamente el $50 \%$. Dichos valores concuerdan con aquellos considerados por Cuyubamba N., en su estudio "Conocimiento y actitudes del personal de salud hacia la aplicación de medidas de bioseguridad" en donde concluye que las actitudes favorable e intermedia en relación a la aplicación de normas de bioseguridad predominan sobre la actitud desfavorable (8). Por lo tanto se evidencia la apertura a desarrollar buenas prácticas respecto a parámetros de bioseguridad.

\section{CONCLUSIONES}

El nivel de conocimiento sobre el concepto de bioseguridad es alto, lo que evidencia la adecuada preparación a nivel cognitivo sobre este parámetro. Asimismo, el nivel de conocimiento acerca del uso de barreras es alto en la mayoría de los casos de acuerdo a cada elemento mencionado, lo cual evidencia la adecuada preparación cognitiva en la mayor parte de los estudiantes. En el caso del manejo de residuos contaminantes, el conocimiento es alto, lo que evidencia que la capacidad cognitiva acerca de este parámetro es adecuada en la mayoría de los casos.

\section{AUTOR DE CORRESPONDENCIA}

Victor Humberto Chero Pacheco

Universidad María Auxiliadora.

Av. Canto Bello No 431

Lima 36 - Perú

Teléfono: +51-1-3891212.

E-mail:vchp_dent@hotmail.com

\section{REFERENCIAS BIBLIOGRÁFICAS}

1. Ministerio de Salud del Perú. Manual de Bioseguridad. Lima: MINSA; 2004.

2. Ministerio de Salud del Perú. Bioseguridad en laboratorios de ensayo, biomédicos y clínicos. Lima: MINSA; 2005.
3. Kumar V., Abbas A., Fausto N., Aster J. Patología estructural y funcional. $8^{\mathrm{a}}$ edición. Barcelona: Elsevier; 2010.

4. Instituto Nacional de Salud del Niño. Manual de bioseguridad. Lima: INSN; 2014.

5. Argente H., Álvarez M. Semiología médica. $2^{\mathrm{a}}$ edición. Buenos Aires: Panamericana; 2013.

6. Lopez R., Lopez M. Nivel de conocimiento y prácticas de medidas de bioseguridad en internos de enfermería del Hospital MINSA II-2 Tarapoto, Junio Agosto 2012 [Tesis]. Tarapoto: Universidad Nacional de San Martín. Facultad de ciencias de la salud; 2012.

7. Sangama L., Rojas R. Nivel de conocimiento y aplicación de medidas de bioseguridad en estudiantes del VIII - IX ciclo de obstetricia UNSM - T en el hospital II-2, Tarapoto. Junio - Setiembre 2012 [Tesis]. Tarapoto: Universidad Nacional de San Martín. Facultad de ciencias de la salud; 2012.

8. Cuyubamba, N. Conocimientos y actitudes del personal de salud hacia la aplicación de medidas de bioseguridad del hospital Félix Mayorca Soto, Tarma 2003 [Tesis]. Lima: Universidad Nacional Mayor de San Marcos. Facultad de Medicina Humana; 2004.

Recibido: 19/08/2016

Aceptado: 04/11/2016 